\title{
CORRESPONDENCE
}

Readers may submit letters to the editor concerning articles that appeared in GASTROENTEROLOGY within one month of publication. Detailed guidelines regarding the content are included in the Instructions to Authors.

\section{The Role of Src Family Kinases in Neuroendocrine Tumors}

Dear Sir:

We read with great interest the paper by Gaur et al, describing the identification of a stem cell population in neuroendocrine tumors (NETs), named by the authors neuroendocrine cancer stem cells (N-CSC), through their ALDH activity as evaluated by the Aldefluor assay. The authors presented convincing evidence about the stemness properties of these cells, as suggested by their in vitro and in vivo self-renewal capacity, particularly by their ability of anchorage-independent growth and tumor growth initiation. They also aimed at characterizing potentially "druggable" pathways in these cells, and found that the Src and mammalian target of rapamycin (mTOR) pathways are active in N-CSC. The authors focused their study on the Src pathway and found that targeting Src inhibited the growth both of $\mathrm{N}$ CSCs cells in vitro, and of tumors derived from them in vivo. The Src pathway has, therefore, been discussed as a potential novel target with therapeutic potential for NETs.

We congratulate with the authors for the excellent work, and we would like to further discuss the potential role of Src family kinases (SFKs) inhibitors for the clinical treatment of NETs.

The US Food and Drug Administration has recently approved both the mTOR inhibitor everolimus, and the multityrosine kinase inhibitor sunitinib, for the treatment of progressive, unresectable, locally advanced or metastatic pancreatic endocrine tumors. ${ }^{2}$ From a molecular viewpoint, the possible limitations of such clinical options include the capacity of cancer cells to develop escape pathways that evoke prosurvival feedback responses, and the existence of cross-talk between different molecular pathways which have not been explored in this cancer type. The activation of escape pathways in tumor cells exposed to targeted therapies has raised interest in the development of combined treatments implying either a "vertical blockage," targeting several steps in the same pathways (ie, PI3K and mTOR, or vascular endothelial growth factor and hypoxia-inducible factors), or a "horizontal blockage" targeting distinct molecular pathways at the same time, which may converge on the same player in the cell. However, there are still very few preclinical studies evaluating these approaches in NETs.

The finding that both mTOR and Src pathways are activated in these N-CSC, recall some recent evidence that suggested a possible link between SFKs activity and the mTOR pathway. ${ }^{3}$ Moreover, it has been previously demonstrated that SFKs are overexpressed in NETs, ${ }^{4}$ and that the inhibition of their activity impairs adhesion, spreading and migration of pancreatic endocrine tumor cells. ${ }^{5}$
In a more recent study, the possible link between the SFK and mTOR pathways in NETs has been further explored, ${ }^{6}$ with findings suggesting a novel role for SFKs in controlling the mTOR activity in pancreatic endocrine tumor cells. In particular, it was found that SFKs couple the event of cell adhesion and spreading with an increase in mTOR activity. Activation of mTOR by SFKs leads to mTOR-dependent activation of translation of a subset of mRNAs for cell-cycle proteins. Moreover, the concomitant inhibition of SFK and mTOR activities strongly impaired NET cell growth, compared with the effect exerted by the single agents. Of note, although treatment with the mTOR inhibitor everolimus triggered the activation of a survival response dependent on PI3K/AKT signaling, the simultaneous inhibition of SFKs blocked the activation of this unwanted escape signal.

We believe that such findings, together with these of Gaur and colleagues, strengthen the interest on the role of Src in NETs, and suggest the need for further preclinical studies with SFK inhibitors in animal models of NETs, and the possible consideration of such drugs for phase I clinical trials in patients with this particular cancer type.

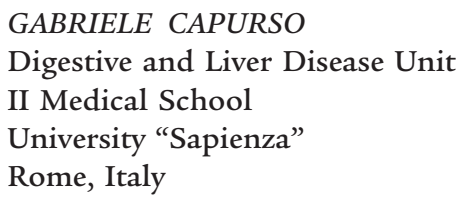

1. Gaur P, et al. Gastroenterology 2011;141:1728-1737.

2. Jensen RT, et al. N Engl J Med 2011;10;364:564-565.

3. Vojtechová M, et al. Neoplasia 2008;10:99-107.

4. Capurso G, et al. Endocr Relat Cancer 2006;13:541-558.

5. Di Florio A, et al. Endocr Relat Cancer 2007;14:111-124.

6. Di Florio A, et al. Endocr Relat Cancer 2011;18:541-554.

\section{Acknowledgements \\ The authors thank Alessia Di Florio for her crucial role in the research projects on Src in NET cells.}

\section{Conflicts of interest}

The authors disclose no conflicts. 